\title{
Comparison with Navigation of a Novel Three- Step Technique for Improving Accuracy of the Distal Femoral Resection during Conventional TKA: A Case-Control Study
}

Arun B. Mullaji, FRCSEd, MChOrth, MSOrth, DNBOrth ${ }^{10}$ Ahmed A. Khalifa, MD, FRCS, MSc ${ }^{2}$ () Gautam Shetty, MS Orth ${ }^{3}$ Harshad Thakur, MBBS, MD, DBM ${ }^{4}$

${ }^{1}$ Department of Orthopaedics, Breach Candy Hospital, Mumbai, Maharashtra, India

2 Department of Orthopaedics, Qena Faculty of Medicine and University Hospital, South Valley University, Qena, Egypt

${ }^{3}$ Mumbai Shoulder and Knee Institute, Brentwood, Powai, Mumbai, Maharashtra, India

${ }^{4}$ National Institute of Health and Family Welfare, Baba Gang Nath Marg, Munirka, New Delhi, India

Address for correspondence Ahmed A. Khalifa, MD, FRCS, MSc, Department of Orthopaedics, Qena University Hospital, South Valley University, Kilo 6 Qena-Safaga Highway, Qena 83523, Egypt (e-mail: ahmed_adel0391@med.svu.edu.eg).

J Knee Surg 2023;36:195-200.

\begin{abstract}
Keywords

- total knee arthroplasty

- valgus correction angle

- distal femur

- alignment

- computer-assisted TKAs

Correct placement of the femoral component in the coronal plane during primary total knee arthroplasty (TKA) is related to long-term survival. The aim of this radiographic study was to determine the accuracy of a novel three-step technique for improving the accuracy of the distal femoral cut during conventional technique and compare it with computer navigation during TKA. A total of 458 TKAs were retrospectively analyzed (178 conventional TKAs with the novel technique and 280 navigated TKAs) for postoperative femoral component coronal alignment and compared between the two groups. Mean femoral component coronal alignment was not significantly different $(p=0.314)$ between the two groups. There was no significant difference in the mean femoral component coronal alignment between varus and valgus knees. The number of outliers ( $90 \pm 3$ degrees) for femoral component coronal alignment was not significantly different between the two groups when assessed separately for varus and valgus deformities. The mean value of femoral component alignment using the conventional technique in knees with varus deformity $<10$ degrees was 88.8 degrees, in knees with varus deformity 10 to 20 degrees was 89.4 degrees, and in those with varus deformity $>20$ degrees was 90.2 degrees. Femoral component alignment in knees with varus $<10$ degrees was significantly different from those $>20$ degrees $(p=0.006)$; there was no significant difference between knees with varus $<10$ degrees and those with 10 to 20 degrees varus $(p=0.251)$, nor between 10 and 20 degrees varus knees and those with varus $>20$ degrees $(p=0.116)$. Using the novel three-step technique during conventional TKA to perform the distal femoral cut can help achieve femoral component coronal alignment comparable to the navigation technique.
\end{abstract}

received

March 12, 2021

accepted after revision

May 1, 2021

article published online

July 8,2021 (c) 2021. Thieme. All rights reserved.

Thieme Medical Publishers, Inc.,

333 Seventh Avenue, 18th Floor,

New York, NY 10001, USA
DOI https://doi.org/

10.1055/s-0041-1731458.

ISSN 1538-8506. 
The survivorship of total knee arthroplasty (TKA) can be affected by factors such as accuracy of bone cuts, limb and component alignment, and soft tissue balancing achieved during surgery. ${ }^{1-3}$ Component malalignment can lead to failure of TKA by causing early polyethylene wear and aseptic loosening; therefore, if the surgeon is aiming for classical mechanical alignment, he would like to align both femoral and tibial components within \pm 3 degrees of neutral with respect to their mechanical axes to facilitate equal distribution of forces across the implant postoperatively. ${ }^{4-7}$

Since the femoral mechanical and anatomical axes are not coincident, a distal femoral cut perpendicular to the coronal femoral mechanical axis is usually achieved by resecting the distal femur using the distal femoral valgus correction angle (VCA), which is equal to the angle between the femoral mechanical and anatomical axes. ${ }^{8-11}$ This cut can be achieved by taking a fixed VCA of 5 to 7 degrees for all cases (which is the standard practice by many surgeons), tailoring the distal cut according to the preoperatively measured VCA, or by using computer-assisted navigation. ${ }^{12,13}$

Several studies have highlighted wide variations in VCA in patients undergoing TKA where it can range from 2 to 13 degrees and how using a fixed VCA range can lead to error in distal femoral cut and femoral component malalignment; hence, it is recommended that the distal femoral cut should be individualized in each patient according to the measured VCA. ${ }^{13}$ However, in a meta-analysis of 29 studies, it was found that the femoral component position is accurately positioned only in $65.9 \%$ of knees within 2 degrees of perpendicular to the mechanical axis in conventional TKAs. $^{14}$

We devised a novel three-step technique that involved radiographically measuring the VCA preoperatively for each knee, using an image intensifier before incision to identify the femoral head center and using an extramedullary verification tool intraoperatively to determine the accuracy of the distal femoral resection. This study hypothesized that the accuracy of distal femoral cut and femoral component place- ment in the coronal plane with this three-step technique, incorporated in the conventional technique of TKA, would be as accurate as computer navigation. To the best of our knowledge, this is the first study in the literature to compare our novel three-step technique with the navigation technique.

\section{Methods}

This is a retrospective radiographic review, performed between February 2019 and October 2019, of the records of 500 consecutive primary TKAs operated by a single surgeon. The inclusion criteria were all patients who underwent primary TKA for osteoarthritis and rheumatoid arthritis and all patients who provided their informed consent for participation in our research study. We excluded patients who had pre- or postoperative radiographs of inappropriate quality or patients whose radiographs were unavailable for evaluation, and patients who were lost to follow-up. The study received institutional ethics committee approval.

All surgeries were performed at either of two centers: the optimized conventional technique of TKA in one center and the navigation technique of TKA in the other center. Hence, patients received either an optimized conventional or navigated TKA depending on which center was selected by the patient (chosen by patients based on the proximity of their geographic place of residence to the surgery center). The study was approved by the ethics committee of the institution. Of the 500 TKAs enrolled during the study period, 42 TKAs were excluded due to the unavailability of radiographs or inappropriate quality of pre- or postoperative radiographs. A total of 458 TKAs were available for radiographic analysis, with 178 TKAs in the optimized conventional group and 280 TKAs in the computer navigation group (-Table $\mathbf{1}$ ). There were 435 varus knees and 23 valgus knees in the study.

Full-length, weight-bearing, hip-to-ankle radiographs were obtained before and within 4 weeks after surgery. The postoperative radiographs were all obtained in the

Table 1 Comparison of independent variables between the two groups

\begin{tabular}{|c|c|c|c|c|}
\hline Independent variables & $\begin{array}{l}\text { Navigated group } \\
(n=280)\end{array}$ & $\begin{array}{l}\text { Novel conventional group } \\
(n=178)\end{array}$ & $\begin{array}{l}\text { Total } \\
(n=458)\end{array}$ & $p$-Value and significance \\
\hline \multicolumn{5}{|l|}{ Gender } \\
\hline Male & 67 (23.9\%) & $41(23.0 \%)$ & $108(23.6 \%)$ & \multirow{2}{*}{$\begin{array}{l}\text { Chi-square test }=0.048 \\
p=0.826, \text { NS }\end{array}$} \\
\hline Female & $213(76.1 \%)$ & $137(77.0 \%)$ & $350(76.4 \%)$ & \\
\hline \multicolumn{5}{|l|}{ Side } \\
\hline Left & 127 (45.4\%) & $91(51.1 \%)$ & $218(47.6 \%)$ & \multirow{2}{*}{$\begin{array}{l}\text { Chi-square test }=1.451 \\
p=0.228, \mathrm{NS}\end{array}$} \\
\hline Right & $153(54.6 \%)$ & $87(48.9 \%)$ & $240(52.4 \%)$ & \\
\hline $\begin{array}{l}\text { Preoperative alignment } \\
\text { (HKA angle in deg) }\end{array}$ & $\begin{array}{l}167.5 \pm 7.8 \\
(138.4-206.5)\end{array}$ & $\begin{array}{l}166.8 \pm 11.1 \\
(126.5-213.0)\end{array}$ & $\begin{array}{l}167.2 \pm 9.2 \\
(126.5-213.0)\end{array}$ & $\begin{array}{l}t=-0.827 \\
p=0.409, \mathrm{NS}\end{array}$ \\
\hline \multicolumn{5}{|l|}{ Type of deformity } \\
\hline Varus & 270 & 165 & 435 & \multirow{2}{*}{$\begin{array}{l}\text { Chi-square test }=3.177 \\
p=0.075, \mathrm{NS}\end{array}$} \\
\hline Valgus & 10 & 13 & 23 & \\
\hline
\end{tabular}

Abbreviations: HKA, hip-knee-ankle; NS, not significant.

Notes: All values are presented as mean \pm standard deviation (minimum-maximum) or number (percentage). $p<0.05$ is considered significant. 
same institution using a standard radiographic protocol (with both knees in full extension, patellae facing forward, and both feet pointing forward to avoid malrotation of the limb during radiography). All full-length radiographs were screened by one of the authors for excessive rotation in the coronal plane which made the radiograph unsuitable for analysis. ${ }^{15}$ The excessive rotation was determined on radiographs by the profile of the lesser trochanter and fibular head and whether the patella was centered or medial/lateral. Digital images of the radiographs were used for measuring various radiographic parameters using the Image J image processing and analysis software (version 1.41, U.S. National Institute of Health). Radiographic parameters measured included pre- and postoperative coronal limb alignment or hip-knee-ankle (HKA) angle defined as the angle between the femoral mechanical axis (line joining the center of the femoral head and center of the knee joint) and the tibial mechanical axis (line joining the center of the knee joint and center of the ankle joint), and the postoperative femoral component coronal alignment defined as the medial angle between the femoral mechanical axis and the line tangential to the prosthetic femoral condyles. All measurements on radiographs were performed by an independent surgeon who was not associated with any of the surgeries and was blinded to the type of technique used.

All procedures were performed under a tourniquet which was deflated when the cement hardened. All knees were approached with a standard medial parapatellar arthrotomy. All patients received a cemented, posterior-stabilized, fixedbearing implant (PFC Sigma, DePuy Inc, Warsaw, IN) with resurfacing of the patella. The same cutting blocks were used for performing the bony resections in both groups. The aim was to achieve a limb coronal mechanical axis of $180 \pm 3$ degrees and position the femoral component perpendicular to the femoral mechanical axis \pm 3 degrees. The degree of soft tissue release was based on the amount of soft tissue tightness assessed using a manual tensioning device and spacer block. Medial release in varus knees and lateral release in valgus knees were performed to achieve rectangular balanced gaps and a fully restored mechanical axis. We used the balanced gap technique, the tibial cut being performed first, and the extension gap being balanced before the flexion gap.

In the patients who had TKAs performed with computer navigation (Brain Laboratory, Munich, Germany), registration was performed in a standard fashion after fixation of infrared reflecting arrays on the distal femur and proximal tibia. The coronal mechanical axis of the lower limb was derived by the navigation software using the center of the femoral head, the center of the intercondylar notch, the center of the tibial plateau, and the center of the ankle plafond. A verification tool with reflecting arrays was used to position the cutting blocks and verify and quantify the distal femoral cut.

In the conventional TKA group, we used a novel three-step method comprising (1) measuring the VCA for each knee on preoperative full-length, hip-to-ankle radiographs. This angle was used to individualize the distal femoral resection

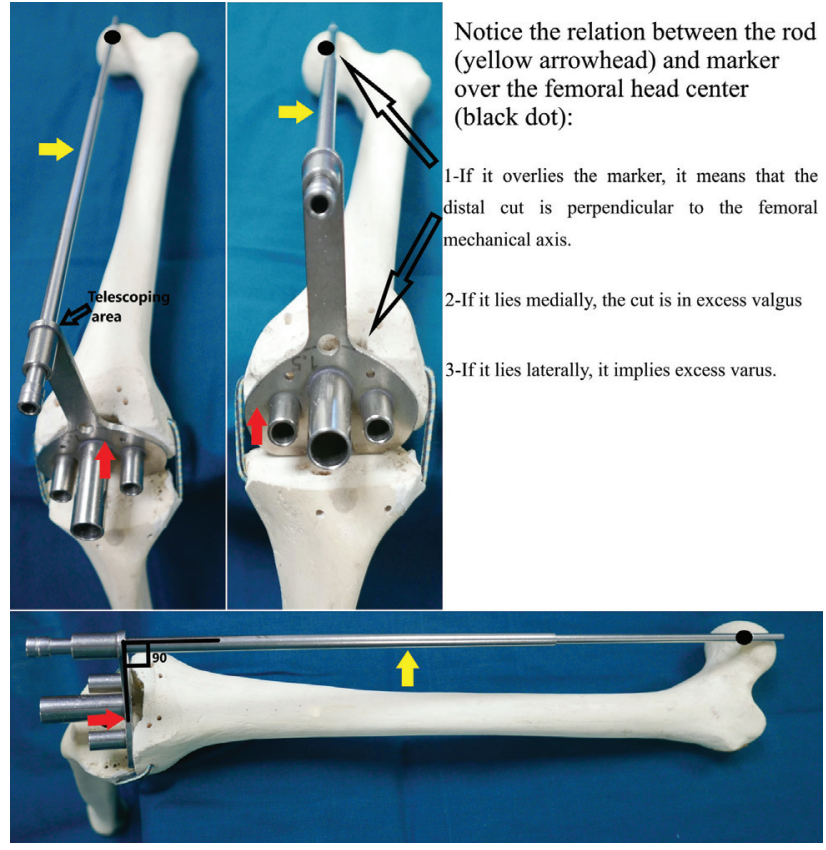

Fig. 1 The extramedullary verification tool comprises a base plate (red arrowhead) that is placed on the resected surface of the distal femoral. The plate is fixed to a vertical arm (yellow arrowhead) with a sleeve, into which is slid a telescoping rod. The relationship of the proximal end of the rod to the marker placed in the groin (not shown, presented by the black dot) is palpated.

in the coronal plane in each patient. Hence, on a full-length radiograph, if the VCA was measured as 8 degrees, the distal femoral resection guide was set at 8 degrees valgus so as to achieve a distal femoral cut perpendicular to the femoral mechanical axis. (2) After the patient was anesthetized, an image intensifier was used to identify the femoral head center, and a radio-opaque adhesive marker was placed in the groin as a surface marker for the femoral head center. (3) During surgery, an extramedullary verification tool, consisting of a long rod which was fixed perpendicular to a flat plate, was used to determine the accuracy of the distal femoral resection (-Fig. 1) (after the individualized cut had been performed using the distal resection block placed with an intramedullary rod) with reference to the previously placed femoral head center radio-opaque marker. Any disparity was corrected by a freehand recut.

Statistical analysis was performed using Microsoft Excel v16.37 and StatPlus2 v 7.1. Data from the two groups of patients were compared using Student's $t$-test, Mann-Whitney's $U$-test, chi-square test, Fisher's exact test, and analysis of variance with Tukey's post hoc test. A significant difference was taken to be $p<0.05$.

\section{Results}

- Table 1 shows that there is no significant difference for all the four independent variables (gender, side, preoperative alignment value [HKA], and type of deformity [varus or valgus]) between navigated and novel conventional groups, implying that all the independent variables were equally distributed between the two groups. 


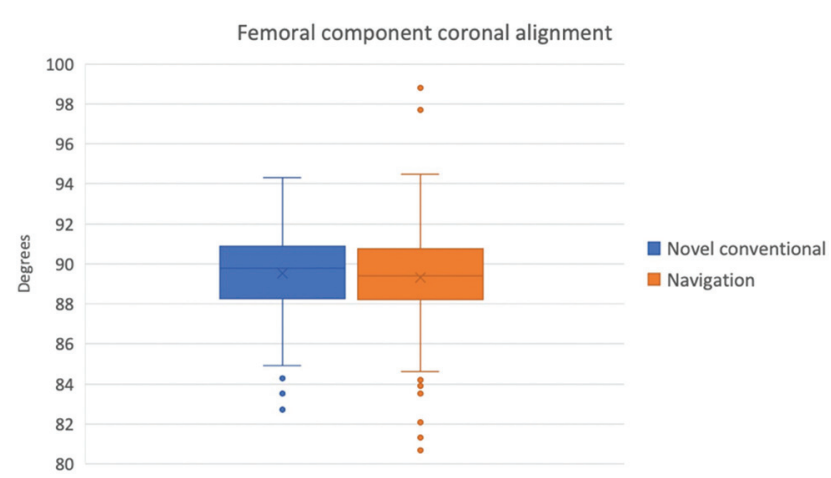

Fig. 2 Box and whisker plot showing femoral component alignment (degrees) in the novel conventional technique and with navigation.

The mean femoral component coronal alignment was 89.3 degrees \pm 2.2 (80.7-98.8 degrees) in the navigated group and 89.5 degrees \pm 2.0 (82.7-94.3 degrees) in the novel conventional group (-Fig. 2). After carrying out an independent $t$-test between the two groups, there was no significant difference $(p=0.314)$.

On excluding the valgus knees from both groups, the mean femoral component coronal alignment was 89.2 degrees \pm 2.2 (80.7-98.8 degrees) in the navigated group and 89.4 degrees \pm 1.9 (82.7-93.8 degrees) in the conventional group. After carrying out an independent $t$-test between the two groups, there was no significant difference $(p=0.294)$.

In varus knees, there were 40 outliers outside the \pm 3 degrees range in the navigated group $(31<87$ degrees, 9 $>93$ degrees) and 21 outliers in the conventional group (17 $<87$ degrees, $4>93$ degrees). There were two outliers among the valgus knees in the conventional group (one $<87$ degrees and one $>93$ degrees), and none in the navigated group (-Fig. 3). Totally, there were 12.9 and $14.3 \%$ outliers with the novel conventional technique and the navigation, respectively. The number of outliers for femoral component coronal alignment outside the \pm 3 degrees range from a neutral alignment of 90 degrees in the coronal plane was not significantly different between the two groups (navigation and novel conventional techniques) when assessed separately both for varus (chi-square statistic $0.3702 ; p$-value 0.543 ) and valgus deformities (Fisher's exact test statistic 0.1779, the result being not significant at $p<0.05$ ).

The mean value of femoral component alignment using the conventional technique in knees with varus deformity $<10$ degrees was 88.8 degrees, in knees with varus deformity 10 to 20 degrees was 89.4 degrees, and in those with varus deformity $>20$ degrees was 90.2 degrees ( - Fig. 4 ). When varus knees in the conventional technique were compared based on their preoperative HKA angles, there was a significant difference on analysis of variance in the mean femoral component coronal alignment between knees with varus deformity < 10 degrees, and varus deformity 10 to 20 degrees, and varus deformity $>20$ degrees ( $F$ statistic $4.824, p=0.009$ ). Tukey's post hoc test identified the group of varus knees $<10$ degrees being significantly different from those $>20$ degrees ( $Q$ statistic $4.381, p=0.006$ ). There was no significant difference between varus knees $<10$ degrees and

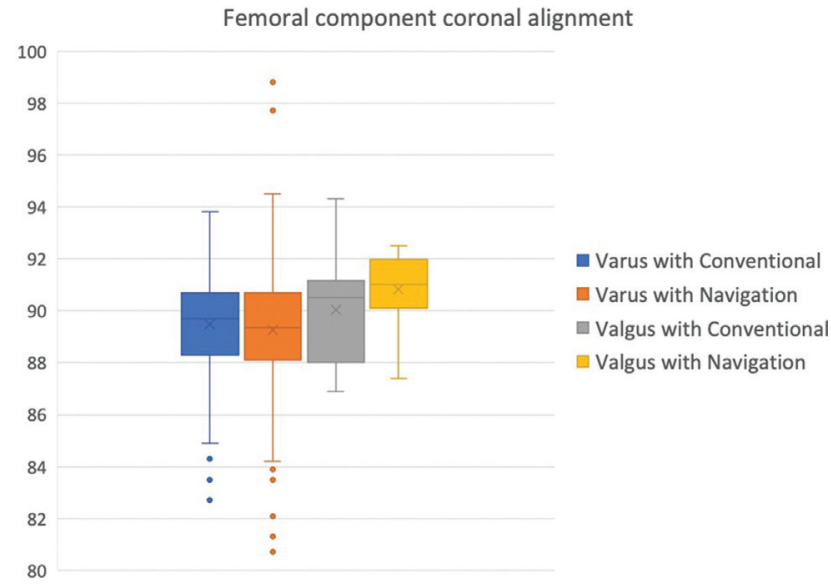

Fig. 3 Box and whisker plot showing femoral component alignment (degrees) separately for knees with preoperative varus and valgus alignment in the novel conventional technique and with navigation.

those with varus deformity 10 to 20 degrees $(p=0.251$ ). There was no significant difference between 10 and 20 degrees varus knees and those with varus knees $>20$ degrees $(p=0.116)$.

Using the Mann-Whitney's $U$-test, there was no significant difference in femoral component alignment between valgus knees in the navigated and novel conventional groups. ( $U$-value $=43$, result not significant at $p<0.05)$.

\section{Discussion}

The main finding of this study was that the mean femoral component coronal alignment was not significantly different when navigation and optimized conventional groups were compared and the percentage of outliers for femoral component coronal alignment outside the \pm 3 degrees range from a neutral alignment of 90 degrees in the coronal plane was not significantly different between the two groups. We found our novel three-step technique of comparable accuracy as computer navigation in improving the accuracy of the distal femoral resection. The technique involved using (1)

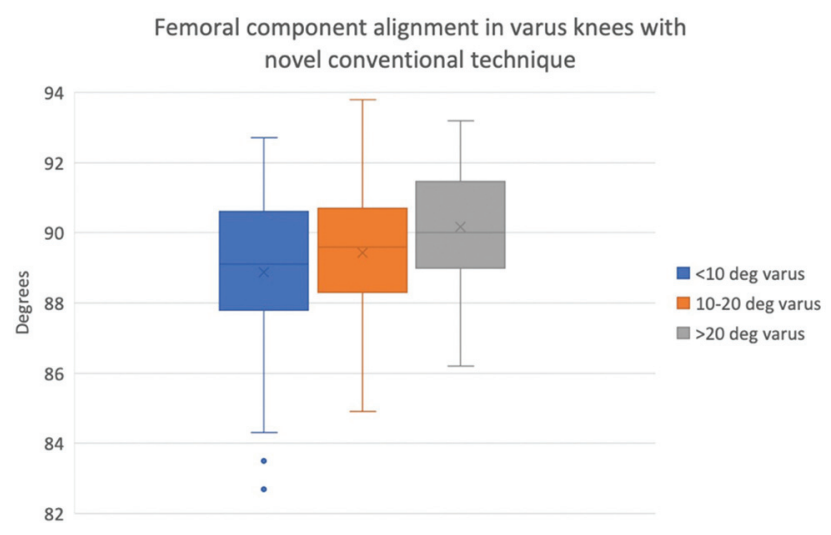

Fig. 4 Box and whisker plot showing femoral component alignment (degrees) for knees with preoperative varus alignment grouped into knees with preoperative varus $<10,10$ to 20 , and $>20$ degrees in the novel conventional technique. 
individualized distal femoral valgus cut according to the measured VCA, (2) markers placed under image intensification to identify the surface position of the hip joint center, and (3) an extramedullary verifying tool. The method of measuring VCA on radiographs is important. Key points to note are as follows: The radiographs must include the entire femur. The limb should be correctly rotated so that the patella is anterior and in the midline. The profile of the lesser trochanter should be just visible. The fibular head should not be obscured by the tibia due to external rotation of the limb.

Also, the distal femoral axis must be a line drawn from the apex of the intercondylar notch to the femur's midpoint at the level (usually middle third-lower third junction in a patient with excess femoral bowing) where the intramedullary rod is likely to engage the canal. In more bowed femora, it may be prudent to use a short rod. The VCA should not be measured using the classical anatomical femoral axis drawn by joining the femoral canal's midpoints at the upper onethird and lower one-third of the femur.

Mason et $\mathrm{al}^{14}{ }^{14}$ in a meta-analysis of 29 studies comparing computer-assisted TKAs to conventional TKAs, reported that $90.4 \%$ of computer-assisted TKAs had femoral component alignment within 2 degrees of perpendicular to the mechanical axis versus $65.9 \%$ in conventional TKAs. However, most of these studies comparing conventional and navigated TKAs used a fixed VCA range of 5 to 7 degrees and have not individualized VCA during TKA which may be the reason why the accuracy of the conventional technique in distal femoral resection was inferior to the navigation technique. Palanisami et al $^{16}$ in a prospective study reported significant improvement in both femoral component placement and postoperative alignment when VCA was individualized when compared with taking a fixed VCA of 5 degrees in patients with moderate and severe varus deformities. Similarly, Shi et al, $^{17}$ in a prospective, randomized study, reported that $83.6 \%$ of knees with femoral component alignment within \pm 3 degrees of the femoral mechanical axis in the individualized VCA group compared with $39.4 \%$ in knees with fixed VCA group.

The VCA shows wide variation among knees, especially with associated extra-articular deformities (such as excessive coronal bowing of the femur) or significant varus deformities., 8 Using a fixed VCA range of 5 to 7 degrees in such patients can cause significant error in distal femoral resection and final femoral implant coronal alignment. Yau et $\mathrm{al}^{12}$ in a study involving Chinese patients reported that at least in 31,31 , or $34 \%$ of the limbs with femoral bowing, a planning error of more than 2 degrees could result if a routine VCA of 5,6 , or 7 degrees was chosen, respectively. Similarly, Mullaji et $\mathrm{al}^{8}$ reported that by choosing a routine VCA of 5,6 , or 7 degrees, the planning error in VCA would be more than 2 degrees in $45.1,28.2$, or $21.1 \%$ of limbs, respectively. An intramedullary rod may be misleading in cases with significant bowing of the femoral shaft or an extraarticular deformity such as a malunited fracture in the distal femur which may cause malalignment of the intramedullary guide rod and distal femoral cutting block. Determining the center of the femur head beforehand using an image intensi- fier in conjunction with using a patient-specific VCA further improves the accuracy of the distal femoral resection, and the extramedullary verification rod affords an opportunity to confirm and fine-tune the precision of the cut.

The mean value of femoral component alignment using the novel conventional technique in knees with varus deformity $<10$ degrees was 88.8 degrees, in knees with varus deformity 10 to 20 degrees $^{\circ}$ was 89.4 degrees, and in those with varus deformity $>20$ degrees was 90.2 degrees. Though analysis of variance showed these three groups to be significantly different, Tukey's post hoc test identified only the group of varus knees $<10$ degrees being significantly different from those $>20$ degrees. However, though statistically significant, the difference of a little over 1 degree is clearly not of clinical significance.

One drawback of the optimized conventional technique is a modest increase by a few minutes in the overall surgical time due to the use of an image intensifier to identify the femoral head center and the ankle joint. This can be minimized by positioning the image intensifier while the patient is being anesthetized. Computer navigation can bypass any femoral extra-articular deformity including excess lateral bowing and directly align the distal femur cutting block perpendicular to the coronal mechanical axis of the femur minimizing the chances of a cutting error. Furthermore, most conventional distal femur cutting guides have a maximum VCA setting of 9 degrees beyond which the surgeon may have to lateralize the entry point which increases the chances of cutting error. In these cases, using computer navigation or the described optimized conventional technique can help improve the accuracy of femoral component placement. However, there is scope for improvement as we still had $12.9 \%$ outliers with femoral component alignment outside the \pm 3 degrees range; that this series included knees with varus even greater than 20 degrees may be borne in mind.

The strengths of this study are the large number of cases, all measured by a standardized technique, by a single independent senior orthopaedic surgeon, and that all surgeries were performed by a single surgeon, using a uniform technique in both groups of patients.

There are some limitations to our study. First, this study has only considered the femoral component placement in the coronal plane. We have not measured femoral component placement in the sagittal plane as full-length lateral sonograms are not performed in our radiology department. Second, since the objective of this study was to compare the accuracy of the femoral component in the coronal plane on full-length radiographs, we have not considered clinical follow-up data regarding functional scores or revision rates. Very often, a compensatory correction may occur deliberately or providentially in the proximal tibial cut. This may help in improving the HKA angle. Occasionally, it may compound the error. Hence, we have not presented the HKA and tibial component values, nor the patient-reported outcome measures. Future follow-up studies regarding the clinical outcome and implant survival in the outliers between the two groups would be valuable. However, the authors believe that the novel three-step modification of the 
optimized conventional technique described in this study will help surgeons using the conventional technique during TKA to achieve accurate placement of the femoral component in knees comparable to navigation. Third, this study involved measuring various parameters on radiographs which is prone to measurement errors. However, limb and component alignment measurements on full-length hip-toankle radiographs have been reported to be reliable and reproducible with good intra- and interobserver correlations. ${ }^{18}$ Fourth, the number of valgus knees was fewer, as valgus deformity is relatively uncommon in the study region. Fifth, we could not include a third group, using a fixed VCA, as several studies have already stated the variability associated with the method. Finally, the study is retrospective.

\section{Conclusion}

In conclusion, using the novel three-step technique during conventional TKA to perform the distal femoral cut can increase the accuracy of femoral component coronal alignment and achieve positioning comparable to that with computer navigation.

\section{Ethical Approval}

This article does not contain any experimental studies with human participants or animals performed by any of the authors. Ethical approval was obtained from the Breach Candy Research Centre ethical committee (project no.: P11/2016). Informed consent was obtained from all individual participants included in the study.

\section{Authors' Contributions}

A.B.M. performed the study conception and design and the critical revision, A.A.K. performed data acquisition and radiographic measurements. G.S., H.T., and A.A.K. performed analysis and interpretation of data, drafted the manuscript, and designed the figures and tables. All authors discussed the results and commented on the manuscript. All authors approved the final manuscript and its appropriateness for submission and publication.

\section{Funding \\ None.}

\section{Conflict of Interest}

None declared.

\section{References}

1 Dyrhovden GS, Fenstad AM, Furnes O, Gøthesen Ø Survivorship and relative risk of revision in computer-navigated versus conventional total knee replacement at 8-year follow-up. Acta Orthop 2016;87(06):592-599
2 Jasper LL, Jones CA, Mollins J, Pohar SL, Beaupre LA. Risk factors for revision of total knee arthroplasty: a scoping review. BMC Musculoskelet Disord 2016;17(01):182

3 Babazadeh S, Stoney JD, Lim K, Choong PF. The relevance of ligament balancing in total knee arthroplasty: how important is it? A systematic review of the literature. Orthop Rev (Pavia) 2009;1(02):e26

4 Thiele K, Perka C, Matziolis G, Mayr HO, Sostheim M, Hube R. Current failure mechanisms after knee arthroplasty have changed: polyethylene wear is less common in revision surgery. J Bone Joint Surg Am 2015;97(09):715-720

5 Valkering KP, Breugem SJ, van den Bekerom MP, Tuinebreijer WE, van Geenen RC. Effect of rotational alignment on outcome of total knee arthroplasty. Acta Orthop 2015;86(04):432-439

6 Srivastava A, Lee GY, Steklov N, Colwell CW Jr, Ezzet KA, D’Lima DD. Effect of tibial component varus on wear in total knee arthroplasty. Knee 2012;19(05):560-563

7 Ritter MA, Davis KE, Meding JB, Pierson JL, Berend ME, Malinzak RA. The effect of alignment and BMI on failure of total knee replacement. J Bone Joint Surg Am 2011;93(17):1588-1596

8 Mullaji AB, Shetty GM, Kanna R, Vadapalli RC. The influence of preoperative deformity on valgus correction angle: an analysis of 503 total knee arthroplasties. J Arthroplasty 2013;28(01):20-27

9 Lee CY, Huang TW, Peng KT, Lee MS, Hsu RW, Shen WJ. Variability of distal femoral valgus resection angle in patients with end-stage osteoarthritis and genu varum deformity: radiographic study in an ethnic Asian population. Biomed J 2015;38(04):350-355

10 Deakin AH, Basanagoudar PL, Nunag P, Johnston AT, Sarungi M. Natural distribution of the femoral mechanical-anatomical angle in an osteoarthritic population and its relevance to total knee arthroplasty. Knee 2012;19(02):120-123

11 Lee DW, Lee J, Lee J, Ro DH, Lee MC, Han HS. A flexible intramedullary guide can reduce the anteroposterior oversizing of femoral components used in total knee arthroplasty in patients with osteoarthritis and severe distal femoral sagittal bowing. J Knee Surg 2021. Doi: 10.1055/s-0040-1722325

12 Yau WP, Chiu KY, Tang WM, Ng TP. Coronal bowing of the femur and tibia in Chinese: its incidence and effects on total knee arthroplasty planning. J Orthop Surg (Hong Kong) 2007;15(01):32-36

13 Nam D, Vajapey S, Haynes JA, Barrack RL, Nunley RM. Does use of a variable distal femur resection angle improve radiographic alignment in primary total knee arthroplasty? J Arthroplasty 2016;31 (09, Suppl):91-96

14 Mason JB, Fehring TK, Estok R, Banel D, Fahrbach K. Meta-analysis of alignment outcomes in computer-assisted total knee arthroplasty surgery. J Arthroplasty 2007;22(08):1097-1106

15 Maderbacher G, Matussek J, Greimel F, et al. Lower limb malrotation is regularly present in long-leg radiographs resulting in significant measurement errors. J Knee Surg 2021;34(01):108-114

16 Palanisami D, Iyyampillai G, Shanmugam S, Natesan RS RIndividualised distal femoral cut improves femoral component placement and limb alignment during total knee replacement in knees with moderate and severe varus deformity. Int Orthop 2016;40 (10):2049-2054

17 Shi X, Li H, Zhou Z, Shen B, Yang J, Pei F. Comparison of postoperative alignment using fixed vs individual valgus correction angle in primary total knee arthroplasty with lateral bowing femur. J Arthroplasty 2016;31(05):976-983

18 Skyttä ET, Haapamäki V, Koivikko M, Huhtala H, Remes V. Reliability of the hip-to-ankle radiograph in determining the knee and implant alignment after total knee arthroplasty. Acta Orthop Belg 2011;77(03):329-335 